\title{
水热法合成氧化亚钴纳米粒子/石墨烯复合材料及其储锂性能研究
}

\author{
王蕾赵冬冬刘旭 于鹏付宏刚* \\ (功能无机材料化学教育部重点实验室 黑龙江大学 哈尔滨 150080)
}

\begin{abstract}
摘要 针对目前的锂离子电池负极材料存在比容量低、循环稳定性差等问题, 本工作发展了简单、有效的方法合成氧 化亚钴纳米粒子与石墨烯的复合材料 $(\mathrm{CoO} / \mathrm{RGO})$. 采用氧化石墨 $(\mathrm{GO})$ 和 $\mathrm{Co}\left(\mathrm{NO}_{3}\right)_{2}$ 作为原料, 先用水热路线制备了前驱 体, 再将其在氮气气氛下热处理, 最终得到 $\mathrm{CoO} / \mathrm{RGO}$ 复合材料. 存在于石墨烯表面的 $\mathrm{CoO}$ 纳米粒子可以有效地阻止石 墨烯片层的聚集, 同时石墨烯片层的相互连接能够形成三维的空间网络, 提高复合材料的导电性. 将合成的 $\mathrm{CoO} / \mathrm{RGO}$ 复合材料作为负极, 以锂片作为正极, 组装成纽扣电池. 电化学测试表明, 在电流密度为 $100 \mathrm{~mA} \cdot \mathrm{g}^{-1}$ 的条件下, 初始比 容量放电比容量高达 $1312.6 \mathrm{mAh} \cdot \mathrm{g}^{-1}$, 在 $10000 \mathrm{~mA} \cdot \mathrm{g}^{-1}$ 的大电流密度下, 经过 300 圈循环后, 其比容量仍然可以达到 $557.4 \mathrm{mAh} \cdot \mathrm{g}^{-1}$. 这表明 $\mathrm{CoO} / \mathrm{RGO}$ 复合材料具有高的比容量、优异的倍率性能及循环稳定性, 这归因于 $3 \mathrm{D}$ 网状结构能 够避免在锂离子的嵌入/脱出过程中材料的结构被严重破坏.
\end{abstract}

关键词 氧化亚钴; 石墨烯; 复合材料; 锂离子电池; 负极材料

\section{Hydrothermal for Synthesis of CoO Nanoparticles/Graphene Composite as Li-ion Battery Anodes}

\author{
Wang, Lei Zhao, Dongdong Liu, Xu Yu, Peng Fu, Honggang* \\ (Key Laboratory of Functional Inorganic Material Chemistry, Ministry of Education of the People's Republic of China, \\ Heilongjiang University, Harbin 150080)
}

\begin{abstract}
Nowadays, the clean energy is of special concern researches owing to the unavoidable environmental pollutions. To satisfy the demand of sustainable development strategy, it is necessary to develop high-efficient and portable energy storage and conversion devices. Lithium ion batteries (LIBs) are considered as most promising electrochemical energy storage system in this era and are anticipated to power the mentioned applications. Herein, a facile and effective route has been developed for synthesis of $\mathrm{CoO}$ /reduced graphite oxide (RGO) composites as LIB anodes. In the synthesis, the GO prepared by the modified Hummers' method was dissolved into deionized water, and then mixed with $\mathrm{Co}\left(\mathrm{NO}_{3}\right)_{2}$ solution. Subsequently, the obtained homogeneous solution was transferred into $100 \mathrm{~mL}$ Teflon-lined stainless-steel autoclave. The sealed autoclave was putted into an oven at $160{ }^{\circ} \mathrm{C}$ for $6 \mathrm{~h}$. After cooled down to room temperature, the precursor of depositions were filtered, washed with deionized water and dried at $80{ }^{\circ} \mathrm{C}$. Finally, the precursor was thermal treated at $500{ }^{\circ} \mathrm{C}$ for $2 \mathrm{~h}$ in a tube furnace under nitrogen ambient to obtain the final product of $\mathrm{CoO} / \mathrm{RGO}$ composites. The synthetic composites were characterized by X-ray diffraction (XRD) and scanning electron microscopy (SEM). XRD patterns proved that the composites were composed of $\mathrm{CoO}$ and graphene. SEM images indicated the $\mathrm{CoO}$ nanoparticles grown on the graphene nanosheets uniformly. The $\mathrm{CoO}$ nanoparticles loaded on the surface of graphene nanosheets could prevent the aggregation of graphene. Meanwhile, the graphene nanosheets could combine with each other to form a large 3D electron conductive network, which can promote the electrical conductivity of the composite. The LIB was assembled in glove-box, in which the composite electrode and metal lithium plate were used as the anode and the cathode, respectively. The electrochemical test results imply that the initial discharge specific capacity could be up to $1312.6 \mathrm{mAh} \bullet \mathrm{g}^{-1}$ at a current density of $100 \mathrm{~mA} \cdot \mathrm{g}^{-1}$. Notably, the discharge specific capacity is still about $557.4 \mathrm{mAh} \bullet \mathrm{g}^{-1}$ after 300 cycles at a high current density of $10000 \mathrm{~mA} \bullet \mathrm{g}^{-1}$. It is demonstrated that the composite exhibits high specific capacity, excellent rate capability and well cyclic stability. The 3D network could be used as a stable framework to accommodate the volume change of active material during $\mathrm{Li}^{+}$insertion/extraction, which play important role for the superior electrochemical performance.
\end{abstract}

Keywords cobalt oxide; graphene; composite; Li-ion batteries; anode materials

\footnotetext{
* E-mail: fuhg@vip.sina.com; Tel.: 0451-86604330

Received September 6, 2016; published January 21, 2017.

Project supported by the National Natural Science Foundation of China (Nos. 21371053, 21401048), application technology research and development projects in Harbin (No. 2013AE4BW051), the international science \& technology cooperation program of China (2014DFR41110), Harbin science and technology innovation talents research Foundation (No. 2015RAQXJ057).

项目受国家自然科学基金(Nos. 21371053, 21401048)、哈尔滨市应用技术研究与开发计划项目(No. 2013AE4BW051)、国家国际科技合作专项项目 (2014DFR41110)和哈尔滨市科技局青年后备人才项目(No. 2015RAQXJ057)资助.
} 


\section{1 引言}

清洁可靠的能源供应方式已成为 21 世纪最重要的 关注点之一, 这是由于它与人们的日常生活、全球的环 境与经济以及人类的健康息息相关 ${ }^{[1 \sim 9]}$. 尽管基于燃烧 的能源技术在满足我们的能源需求方面一直占据主导 地位, 但是其仍然存在增加温室气体排放量以及环境污 染等问题. 即将面临的化石燃料的短缺以及日益增长的 环境问题促使科研工作者积极探索和利用可持续、清洁 和高效的技术来进行能量的供应和存储 ${ }^{[10 \sim 16]}$.

锂离子电池作为重要的电力能源存储设备, 已经引 起了全世界的研究兴趣 ${ }^{[17 ~ 27]}$. 目前, 锂离子电池通常采 用廉价的石墨为负极材料 ${ }^{[28 ~ 32]}$. 但是其存在理论比容 量小 $\left(372 \mathrm{mAh} \cdot \mathrm{g}^{-1}\right)$ 、首次放电效率低且稳定性较差等问

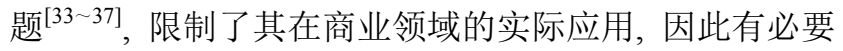
开发高性能电极材料. 石墨烯是一种新型的二维材料, 具有优异的导电性及特殊的纳米片层结构 ${ }^{[38 \sim 41]}$. 石墨 烯是通过氧化石墨(GO) 高温加热制得, 氧化石墨烯表 面具有大量的含氧基团、羟基、环氧官能团, 这些含氧 官能团的存在使得氧化石墨烯表面带有负电荷, 有利于 石墨烯与氧化物之间的复合. 具有不同尺寸及形貌的过 渡金属氧化物与石墨烯的复合材料已被广泛应用于锂 离子电池负极材料 ${ }^{[42 ~ 45]}$. 由于协同作用与界面相互作 用的结果, 石墨烯与氧化物的复合材料可以改善电池的 充放电比容量、倍率特性以及循环稳定性.

本研究工作中我们制备了 $\mathrm{CoO}$ 和石墨烯(RGO)的 复合材料, 并研究了其储锂性能. 首先采用 Hummers 法 制备了 $\mathrm{GO}$, 将其在水中分散均匀再与 $\mathrm{Co}\left(\mathrm{NO}_{3}\right)_{2}$ 溶液混 合. 先采用水热法制备了前驱体, 再将得到的前驱体在 氮气气氛下进行热处理, 最终得到 $\mathrm{CoO} / \mathrm{RGO}$ 复合材料. 作为锂电负极, 展现了较高的比容量、优异的倍率性能 及循环稳定性, 这源自于 $\mathrm{CoO}$ 和 $\mathrm{RGO}$ 之间的协同效应 及界面作用. 该材料在锂离子电池负极材料中具有潜在 的应用价值.

\section{2 结果与讨论}

\section{$2.1 \mathrm{CoO} / \mathrm{RGO}$ 复合材料的结构表征}

$\mathrm{X}$ 射线衍射 (XRD)是表征材料晶相组成的有效手 段. $\mathrm{CoO} / \mathrm{RGO} 1, \mathrm{CoO} / \mathrm{RGO} 2, \mathrm{CoO} / \mathrm{RGO} 3$ 三个复合材料 的 XRD 如图 1 所示. 其中, 衍射角 $2 \theta$ 在 $42.3^{\circ}$ 和 $44.5^{\circ}$ 的两个峰为 $\mathrm{CoO}$ 晶相的特征衍射峰, 衍射角 $2 \theta$ 在 $25^{\circ}$ 左右的宽峰为石墨烯(RGO)的特征衍射峰, 这表明我们 成功合成了 $\mathrm{CoO} / \mathrm{RGO}$ 复合材料. 为了研究石墨烯的存 在对复合材料储锂性能的影响, 我们在制备过程中没有 加入氧化石墨(GO), 得到的是 $\mathrm{Co}_{3} \mathrm{O}_{4}$ 晶相, 并且具有较 好的结晶度(如图 1 所示). 这是由于 $\mathrm{GO}$ 的还原作用, 导 致在合成过程中加入 $\mathrm{GO}$ 时钴的晶相由 $\mathrm{Co}_{3} \mathrm{O}_{4}$ 向 $\mathrm{CoO}$ 转 变.

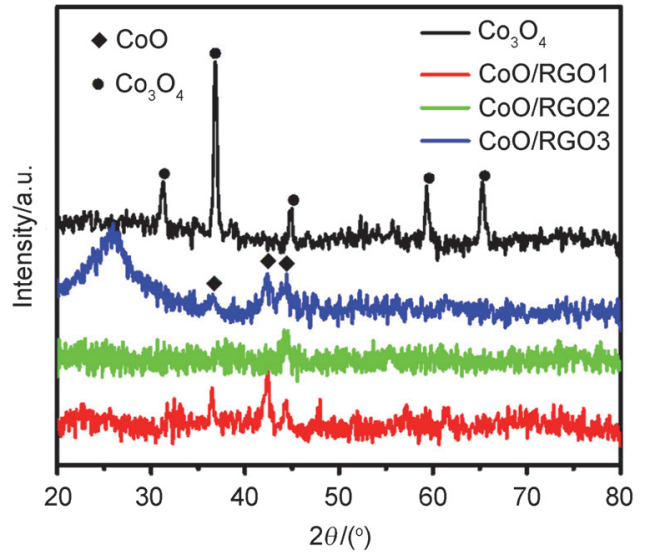

图 $1 \mathrm{Co}_{3} \mathrm{O}_{4}, \mathrm{CoO} / \mathrm{RGO} 1, \mathrm{CoO} / \mathrm{RGO} 2$ 和 $\mathrm{CoO} / \mathrm{RGO} 3$ 样品的 $\mathrm{XRD}$ 图 Figure 1 XRD patterns of $\mathrm{Co}_{3} \mathrm{O}_{4}, \mathrm{CoO} / \mathrm{RGO} 1, \mathrm{CoO} / \mathrm{RGO} 2$ and $\mathrm{CoO} / \mathrm{RGO} 3$ samples

扫描电子显微镜(SEM)用于表征样品的微观形貌及 结构. 图 2 为 $\mathrm{Co}_{3} \mathrm{O}_{4}, \mathrm{CoO} / \mathrm{RGO} 1, \mathrm{CoO} / \mathrm{RGO} 2$, $\mathrm{CoO} / \mathrm{RGO} 3$ 样品的 SEM 图. 如图 2(A)所示, 纯的 $\mathrm{Co}_{3} \mathrm{O}_{4}$ 呈现了表面具有丰富孔的薄片结构. 然而对于 $\mathrm{CoO} / \mathrm{RGO}$ 复合材料来说, 如图 3(B D) 所示, 石墨烯呈 现了聚集的块状结构, 并且随着 GO 量的增加这种团聚 现象更加明显, 这主要是由于高温热处理导致石墨烯片 层团聚. 另外, 可以看出 $\mathrm{CoO}$ 纳米粒子与 $\mathrm{RGO}$ 紧密接 触, 这有利于电子的快速传输, 使其储锂性能提高. 透 射电子显微镜(TEM) 可以用于进一步分析样品的微观结 构. 由图 3 的 TEM 照片可以看出, $\mathrm{CoO}$ 纳米粒子分散在 石墨烯片层表面, 与石墨烯片层紧密接触, 这有利于离 子电子的传输, 使其储锂性能提高.
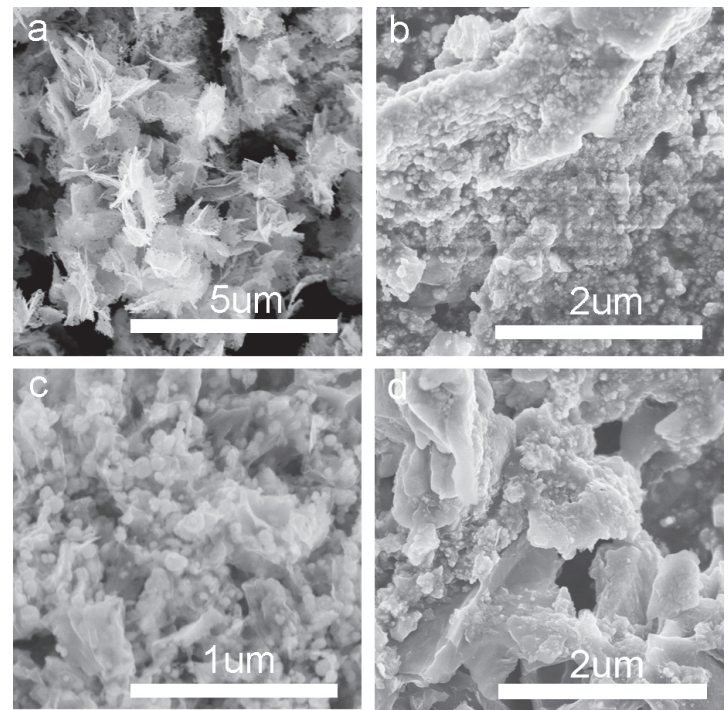

图 2 样品的扫描电子显微镜照片: (A) $\mathrm{Co}_{3} \mathrm{O}_{4}$, (B) $\mathrm{CoO} / \mathrm{RGO} 1$, (C) $\mathrm{CoO} / \mathrm{RGO} 2$, (D) $\mathrm{CoO} / \mathrm{RGO} 3$

Figure 2 SEM images of (A) $\mathrm{Co}_{3} \mathrm{O}_{4}$, (B) $\mathrm{CoO} / \mathrm{RGO}$, (C) $\mathrm{CoO} / \mathrm{RGO} 2$ and (D) $\mathrm{CoO} / \mathrm{RGO} 3$ 


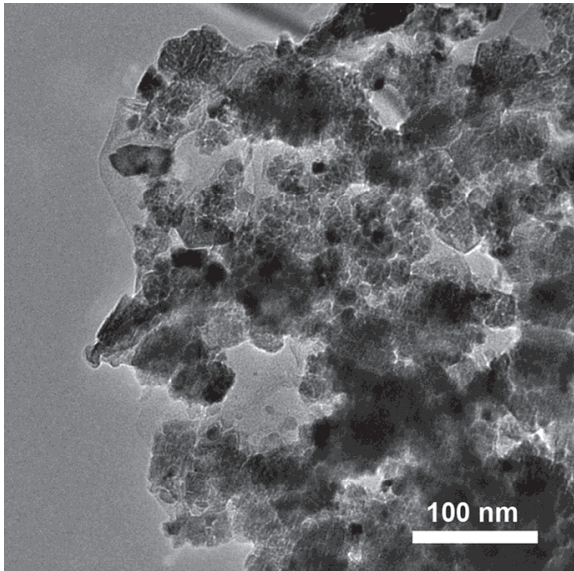

图 $3 \mathrm{CoO} / \mathrm{RGO} 2$ 样品的透射电子显微镜照片

Figure 3 TEM image of $\mathrm{CoO} / \mathrm{RGO} 2$ sample

\section{$2.2 \mathrm{CoO} / \mathrm{RGO}$ 复合材料的电化学性能研究}

由于石墨烯与金属氧化物之间的协同效应，金属氧 化物与石墨烯的复合材料已经被广泛应用于锂离子电 池负极材料中. 为了对材料的储锂性能进行测试, 分别 将本实验制得的复合材料 $\mathrm{CoO} / \mathrm{RGO} 1, \mathrm{CoO} / \mathrm{RGO} 2$, $\mathrm{CoO} / \mathrm{RGO} 3$ 以及 $\mathrm{Co}_{3} \mathrm{O}_{4}$ 作为负极, 锂片作为正极, 将其 组装成纽扣电池, 对其进行了恒电流充放电测试. 图 $4 \mathrm{~A}$ 表明在电流密度为 $100 \mathrm{~mA} \cdot \mathrm{g}^{-1}$ 的条件下, $\mathrm{CoO} / \mathrm{RGO} 2$
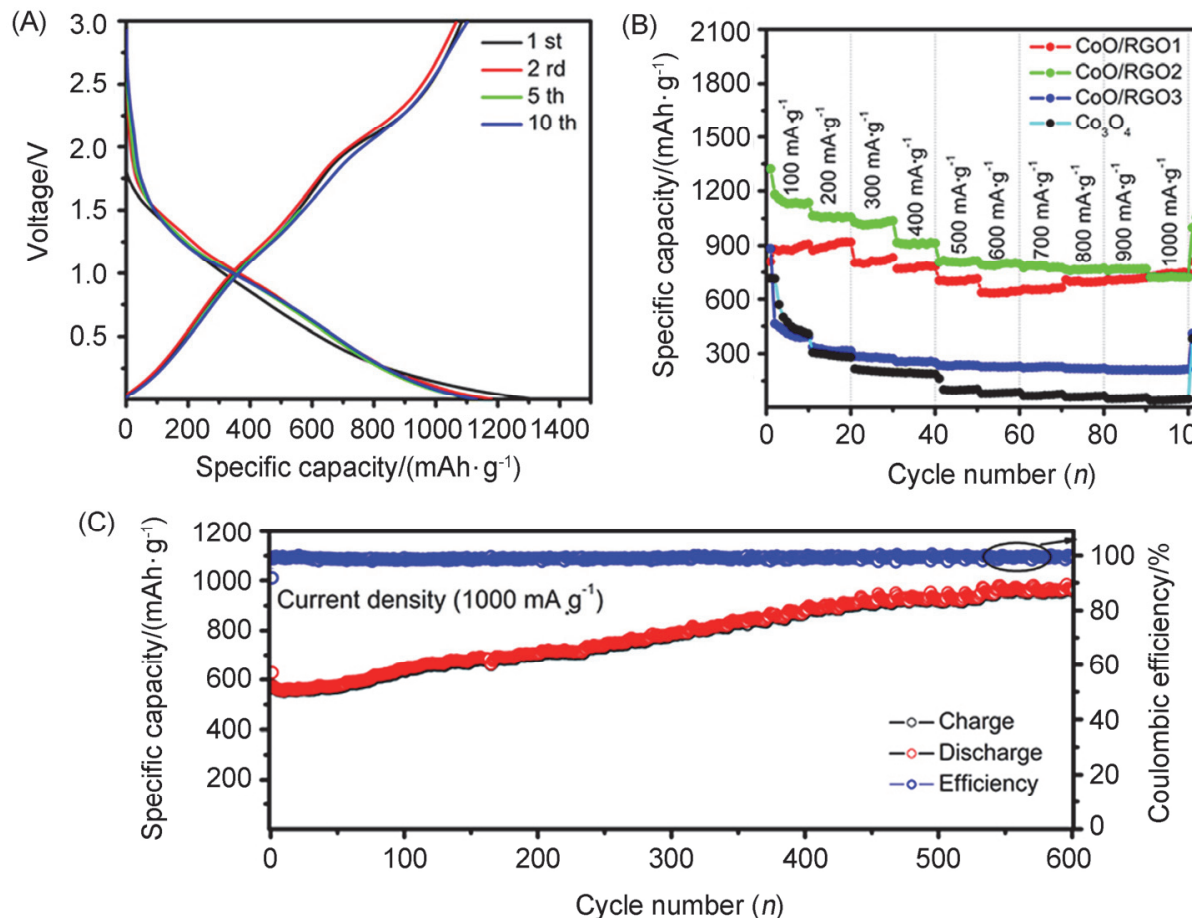

图 4 样品的电化学性能图: (A)电流密度为 $100 \mathrm{~mA} \cdot \mathrm{g}^{-1}$ 时, $\mathrm{CoO} / \mathrm{RGO} 2$ 电极的充放电曲线; (B) $\mathrm{CoO} / \mathrm{RGO} 1, \mathrm{CoO} / \mathrm{RGO} 2, \mathrm{CoO} / \mathrm{RGO}_{3}$ 以及 $\mathrm{Co} \mathrm{O}_{4}$ 电 极在电流密度为 $100 \sim 1000 \mathrm{~mA} \cdot \mathrm{g}^{-1}$ 的比容量变化; (C)在电流密度为 $1000 \mathrm{~mA} \cdot \mathrm{g}^{-1}$ 时 $\mathrm{CoO} / \mathrm{RGO}$ 电极循环 600 圈时的电压-比容量图

Figure 4 Electrochemical performances of the synthetic samples: (A) Charge-discharge profiles for CoO/RGO2 electrode at a current density of $100 \mathrm{~mA}$ $\mathrm{g}^{-1}$; (B) Rate capability for $\mathrm{CoO} / \mathrm{RGO} 1, \mathrm{CoO} / \mathrm{RGO} 2, \mathrm{CoO} / \mathrm{RGO} 3$ and $\mathrm{Co}_{3} \mathrm{O}_{4}$ electrodes at different current density ranging from 100 to $1000 \mathrm{~mA} \cdot \mathrm{g}^{-1}$. (C) Cyclic performance for $\mathrm{CoO} / \mathrm{RGO} 2$ electrode at current densities of $1000 \mathrm{~mA} \cdot \mathrm{g}^{-1}$ for 600 cycles 
电池的倍率性能是评价其性能的重要标准之一, 图 5 为 $\mathrm{CoO} / \mathrm{RGO} 2$ 电极在大电流放电速率时比容量随循环 次数的变化图. 由图 $5 \mathrm{~A}$ 不同电流密度下的比容量变化 可以看出, 随着电流密度由 $2000 \mathrm{~mA} \cdot \mathrm{g}^{-1}$ 向 $50000 \mathrm{~mA} \cdot$ $\mathrm{g}^{-1}$ 逐渐增加, $\mathrm{CoO} / \mathrm{RGO} 2$ 电极展现了优异的倍率特性. 在电流密度为 $2000 \mathrm{~mA} \cdot \mathrm{g}^{-1}$ 时, 其放电和充电比容量分 别为 615.8 和 $619.9 \mathrm{mAh} \cdot \mathrm{g}^{-1}$, 库伦效率为 $99.3 \%$. 即使 是在 $50000 \mathrm{~mA} \cdot \mathrm{g}^{-1}$ 的高电流密度下, 其放电和充电比 容量分别可以达到 216.9 和 $216.2 \mathrm{mAh} \cdot \mathrm{g}^{-1}$, 库伦效率为 $99.9 \%$ 以上. 当电流密度再降至 $2000 \mathrm{~mA} \cdot \mathrm{g}^{-1}$ 时, 其放电 和充电比容量分别为 618.8 和 $616.3 \mathrm{mAh} \cdot \mathrm{g}^{-1}$, 库伦效率 为 $99.6 \%$. 说明 $\mathrm{CoO} / \mathrm{RGO} 2$ 电极的可逆容量较高. 在 $10000 \mathrm{~mA} \cdot \mathrm{g}^{-1}$ 的大电流密度下测试其循环性能, 如图 $5 \mathrm{~B}$ 所示, 随着循环次数的增加其放电和充电比容量是 逐渐增加的, 当循环达到 270 圈以后, 比容量趋于稳定, 最终稳定在 $557.4 \mathrm{mAh} \cdot \mathrm{g}^{-1}$. 此外, 可以看出在循环测试 的过程中, 库伦效率一直保持在 $98 \% \sim 99.9 \%$, 说明 $\mathrm{CoO} / \mathrm{RGO} 2$ 电极具有较好的循环稳定性及高的可逆性.
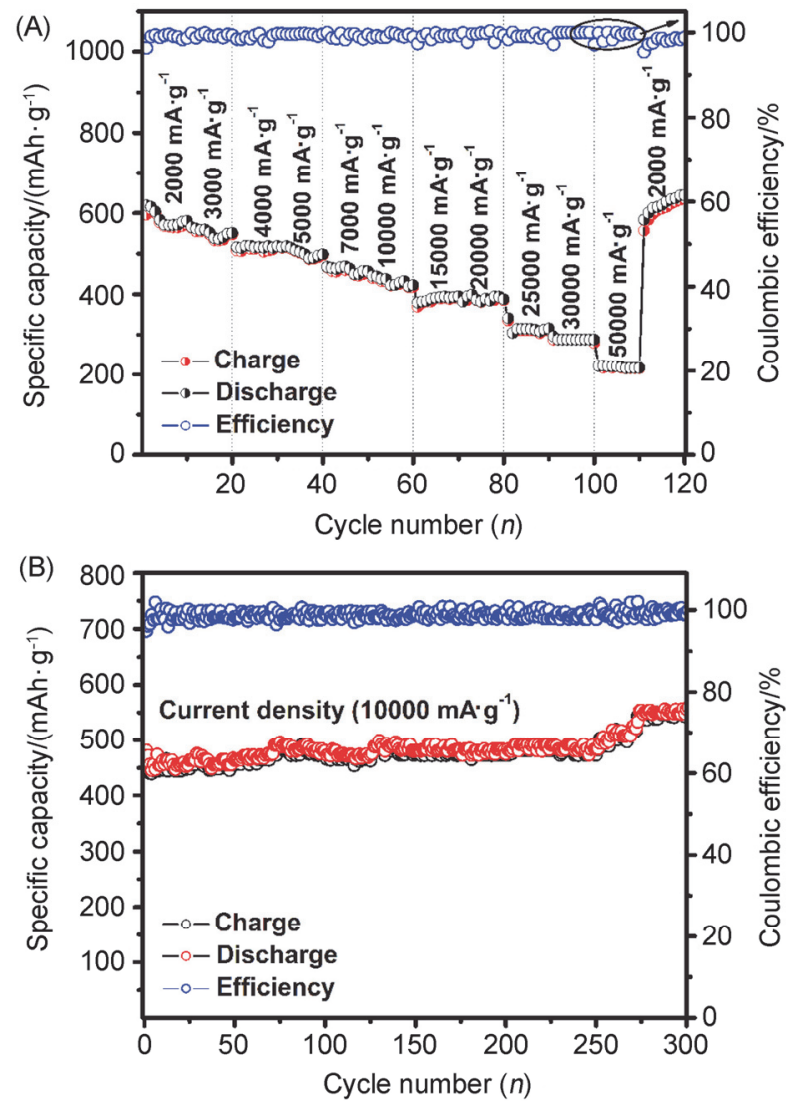

图 $5 \mathrm{CoO} / \mathrm{RGO} 2$ 的电化学性能图: (A)大电流密度下 $(2000 \sim 50000$ $\mathrm{mA} \cdot \mathrm{g}^{-1}$ ) 的比容量与循环圈数的关系; (B) 电流密度 $10000 \mathrm{~mA} \cdot \mathrm{g}^{-1}$ 下 的循环稳定性及库伦效率 $(\approx 99 \%)$

Figure 5 (A) Rate capability at different current density ranging from 2000 to $50000 \mathrm{~mA} \cdot \mathrm{g}^{-1}$; (B) Cycling performance at current densities of $10000 \mathrm{~mA} \cdot \mathrm{g}^{-1}$ with Coulombic efficiency of $99 \%$
电池的电化学阻抗谱图(EIS)及循环伏安测试如图 6 所示. 图 6A 可以看出所有的 EIS 谱图均包括一个高频 区域的半圆形和低频区域的斜线. 高频区域的半圆形主 要包括界面电荷传递阻抗 $\left(R_{\mathrm{ct}}\right)$ 和 $\mathrm{SEI}$ 膜阻抗 $\left(R_{\mathrm{SEI}}\right)$, 而 低频区域的斜线主要归因于锂离子的扩散阻抗. 半圆形 的半径越小、斜率越大，说明锂离子能够更快地扩散传 输通过 SEI 膜、加快电荷转移的反应速率. 从图中可以 明显地看出, 与复合材料相比, $\mathrm{Co}_{3} \mathrm{O}_{4}$ 具有更低的界面 电荷传递阻抗和 SEI 阻抗, 这一现象是由于氧化石墨 很难被完全还原，还原后的石墨烯表面仍然具有很多含 氧基团，导致复合物的导电性并没有纯的 $\mathrm{Co}_{3} \mathrm{O}_{4}$ 好. 但 是由于纯的 $\mathrm{Co}_{3} \mathrm{O}_{4}$ 其没有与石墨烯复合, 因此在电化学 测试中稳定性较差. 在氧化物与石墨烯的复合材料中, $\mathrm{CoO} / \mathrm{RGO} 2$ 样品的阻值介于 $\mathrm{CoO} / \mathrm{RGO} 1$ 和 $\mathrm{CoO} / \mathrm{RGO} 3$ 之间, 又由于其适当的 $\mathrm{CoO}$ 含量, 因此 $\mathrm{CoO} / \mathrm{RGO} 2$ 复合 材料具有优异的倍率性能和循环稳定性. 图 $6 \mathrm{~B}$ 的循环 伏安可以看出在扫速为 $0.1 \mathrm{mV} \cdot \mathrm{s}^{-1}$ 的条件下, $\mathrm{CoO} / \mathrm{RGO} 2$ 复合材料充放电循环 $1 \sim 5$ 圈时, 其循环伏

(A)

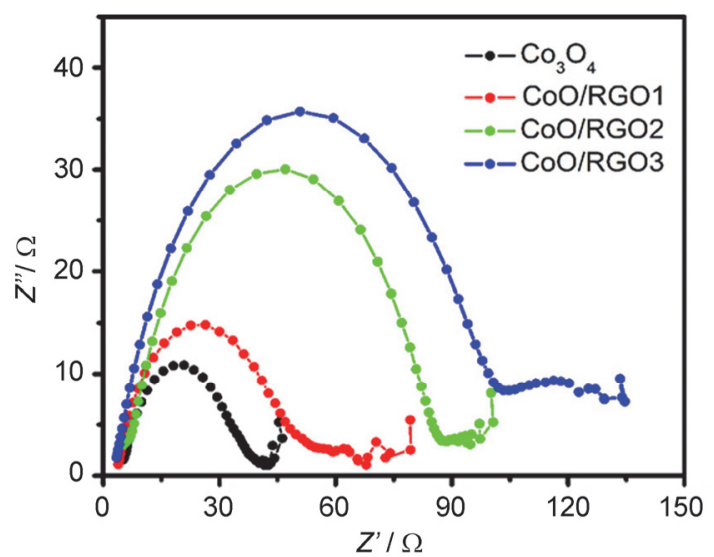

(B)

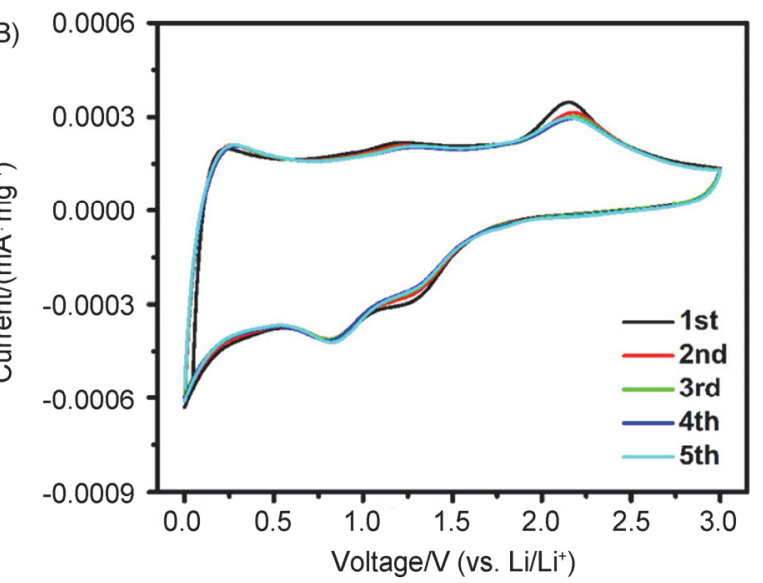

图 6 (A) $\mathrm{Co}_{3} \mathrm{O}_{4}, \mathrm{CoO} / \mathrm{RGO} 1, \mathrm{CoO} / \mathrm{RGO} 2, \mathrm{CoO} / \mathrm{RGO} 3$ 电极的交流阻抗 谱图(EIS); (B) $\mathrm{CoO} / \mathrm{RGO} 1$ 样品的循环伏安图, 电压范围在 $0.01 \sim 3.0$ $\mathrm{V}$, 扫速为 $1 \mathrm{mV} \cdot \mathrm{s}^{-1}$

Figure 6 (A) Nyquist plots of $\mathrm{Co}_{3} \mathrm{O}_{4}, \mathrm{CoO} / \mathrm{RGO} 1, \mathrm{CoO} / \mathrm{RGO} 2$ and $\mathrm{CoO} / \mathrm{RGO} 3$ electrodes; (B) $\mathrm{CVs}$ of $\mathrm{CoO} / \mathrm{RGO} 1$ electrode scanned between $0.01 \sim 3.0 \mathrm{~V}\left(\mathrm{vs} . \mathrm{Li}^{+} / \mathrm{Li}\right)$ at a scan rate of $1 \mathrm{mV} \cdot \mathrm{s}^{-1}$ 
安曲线几乎重合, 这进一步证明了其具有极好的循环稳 定性. 石墨烯基复合材料是一种层层自组装的纳米结 构, 这种结构更加有利于电子和离子的运输, 能够促进 反应的快速进行. 更重要的是, $\mathrm{CoO}$ 与石墨烯的紧密接 触能够扩大活性物质的接触面积以及活性位点, 因此使 复合材料的储锂性能有了很大的提高. $\mathrm{Co}_{3} \mathrm{O}_{4}$ 具有最低 的界面电荷传递阻抗, 但倍率性能却最差, 而加入石墨 烯的三组样品随着石墨烯含量的增加, 界面电阻反而增 大.

\section{3 结论}

总之, 我们采用简单的一步法成功制备了 $\mathrm{CoO} / \mathrm{RGO}$ 复合材料作为锂离子电池的负极材料. $\mathrm{CoO}$ 纳米粒子与石墨烯片层之间的有效复合，使该材料具有 比容量高、倍率性能好及循环稳定性好的特点. 在 $\mathrm{CoO} / \mathrm{RGO}$ 复合材料中, 石墨烯片层能够形成空间的导 电网络从而提高复合材料的电子传导特性. 又由于其具 有优异的机械性能, 因此石墨烯的存在能够起到稳定材 料的结构、提高材料循环稳定性的作用. 另外, 石墨烯 片层与片层之间的间隙有利于电解液的充分浸润, 能够 使活性物质与电解液充分接触, 从而可以有效缩短 $\mathrm{Li}^{+}$ 的传输距离. 本研究工作提供了一种简单、有效的方法 制备高性能锂离子电池负极材料, 为降低锂离子电池的 成本提供了新思路.

\section{4 实验部分}

\subsection{CoO/RGO 复合材料的制备}

称取一定量的石墨微粉, 采用改进的 Hummers 法 制备氧化石墨(GO), 酸洗三次、水洗至中性, 烘干备用. 准确称取 $10 \mathrm{mg} \mathrm{GO}$ 加入到 $50 \mathrm{~mL}$ 去离子水中, 超声分 散均匀后, 倒入 $30 \mathrm{~mL}$ 浓度为 $0.1 \mathrm{~mol} / \mathrm{L}$ 的 $\mathrm{Co}\left(\mathrm{NO}_{3}\right)_{2}$ 溶 液中, 搅拌 $30 \mathrm{~min}$. 将上述混合溶液倒入 $100 \mathrm{~mL}$ 反应 釜中, 在 $160{ }^{\circ} \mathrm{C}$ 温度下反应 $6 \mathrm{~h}$. 反应完成后冷却至室 温后抽滤, 将得到的固体粉末用去离子水洗三次、无水 乙醇洗一次, 随后将其置于 $80{ }^{\circ} \mathrm{C}$ 烘箱中进行干燥处理. 然后在氮气气氛下以 $5{ }^{\circ} \mathrm{C} \cdot \mathrm{min}^{-1}$ 的升温速率加热到 $500{ }^{\circ} \mathrm{C}$, 并恒温 $2 \mathrm{~h}$ 制备了 $\mathrm{CoO} / \mathrm{RGO} 1$ 复合物. 为了研 究 $\mathrm{GO}$ 的用量对样品结构和性能的影响, 我们将 $\mathrm{GO}$ 的 用量调节为 $20 \mathrm{mg}$ 和 $30 \mathrm{mg}$, 其它合成条件不变, 分别 制备了 $\mathrm{CoO} / \mathrm{RGO} 2$ 和 $\mathrm{CoO} / \mathrm{RGO} 3$ 两个样品.

\section{2 材料表征}

采用 X射线粉末衍射仪(XRD)对样品的晶相组成进 行分析, 仪器型号为德国布鲁克公司生产的 Bruker D8 型 X-射线衍射仪, 检测器为 LynxEye 型号, 测试条件为 $\mathrm{Cu} \mathrm{K \alpha}(\lambda=1.5406 \AA)$. 采用扫描电子显微镜(SEM)观察 样品的微观形貌和结构, 测试的加速电压为 $5 \mathrm{kV}$, 仪器 型号为 HitachiS-4800. 采用透射电子显微镜(TEM)观察
样品的微观结构, 测试的加速电压为 $200 \mathrm{kV}$, 仪器型号 为日本电子公司生产的 JEM-2100.

\section{3 电池组装及测试}

将实验所需的正负极外壳、垫片、弹簧片加入到乙 醇中, 在超声频率为 $10 \mathrm{kHz}$ 、超声功率为 $400 \mathrm{~W}$ 条件下, 进行充分的洗涤, 超声时间为 $3 \sim 4 \mathrm{~h}$, 待样品洗净后将 乙醇倒出, 再用乙醇洗涤两次, 随后将其置于 $80{ }^{\circ} \mathrm{C}$ 烘 箱中进行干燥处理, 待冷却至室温后送入真空手套箱 中. $\mathrm{CoO} / \mathrm{RGO}$ 复合材料与乙炔黑、聚偏二氟乙烯(PVDF) 按照 $8: 1: 1$ 的质量比混合均匀, 再加入数滴有机溶剂 NMP ( $N$-甲基-吡咯烷酮), 充分搅拌成均匀糊状物后, 将其均匀涂覆于铜箔上, 在 $80{ }^{\circ} \mathrm{C}$ 下真空干燥 $12 \mathrm{~h}$. 将 涂有 $\mathrm{RGO} / \mathrm{CoO}$ 复合材料的铜䇴为负极, 金属锂片为正 极, 六氟磷锂溶液 [乙烯碳酸酯 (EC) : 碳酸甲乙酯 $(\mathrm{EMC})$ : 碳酸二甲酯 $(\mathrm{DMC})=1: 1: 1$ (体积比) $]$ 为电解 液. 在充满高纯氩气的手套箱内, 将其组装成扣式电池, 随后使用封口机将电池密封好. 待电池静置 $12 \mathrm{~h}$ 后, 用 CT2001A 蓝电电池测试仪对扣式电池进行恒流充放电 性能测试，电压范围为 $0.01 \sim 3.00 \mathrm{~V}$. 用 Autolab PGSTAT302N 电化学工作站进行循环伏安和交流阻抗 测试.

\section{References}

[1] Nishi, Y. Chem. Rec. 2001, 1, 406.

[2] Choi, N.-S.; Chen, Z.; Freunberger, S. A.; Ji, X.; Sun, Y.-K.; Amine, K.; Yushin, G.; Nazar, L. F.; Cho, J.; Bruce, P. G. Angew. Chem. Int. Ed. 2012, 51, 9994.

[3] Cabana, J.; Monconduit, L.; Larcher, D.; Rosa Palacin, M. Adv. Mater. 2010, 22, E170.

[4] Lu, Y.; Wen, Z.; Rui, K.; Wu, X.; Cui, Y. J. Power Sources 2013, $244,306$.

[5] Li, C.; Yin, C.; Mu, X.; Maier, J. Chem. Mater. 2013, 25, 962.

[6] Rui, K.; Wen, Z.; Lu, Y.; Shen, C.; Jin, J. ACS Appl. Mater. Interfaces 2016, 8, 1819.

[7] Li, H.; Liang, M.; Sun, W.; Wang, Y. Adv. Funct. Mater. 2016, 26, 1098.

[8] Wang, X.; Liu, B.; Hou, X.; Wang, Q.; Li, W.; Chen, D.; Shen, G. Nano Res. $2014,7,1073$.

[9] Arun, N.; Jain, A.; Aravindan, V.; Jayaraman, S.; Ling, W. C.; Srinivasan, M. P.; Madhavi, S. Nano Energy 2015, 12, 69.

[10] Arun, N.; Aravindan, V.; Ling, W. C.; Madhavi, S. J. Power Sources 2015, 280, 240.

[11] Han, J.-T.; Goodenough, J. B. Chem. Mater. 2011, 23, 3404.

[12] Lu, X.; Jian, Z.; Fang, Z.; Gu, L.; Hu, Y.-S.; Chen, W.; Wang, Z.; Chen, L. Energy Environ. Sci. 2011, 4, 2638.

[13] Guo, B.; Yu, X.; Sun, X.-G.; Chi, M.; Qiao, Z.-A.; Liu, J.; Hu, Y.-S.; Yang, X.-Q.; Goodenough, J. B.; Dai, S. Energy Environ. Sci. 2014, 7, 2220.

[14] Tang, K.; Mu, X.; PvanAken, A.; Yu, Y.; Maier, J. Adv. Energy Mater. 2013, 3, 49 .

[15] Han, J.-T.; Huang, Y.-H.; Goodenough, J. B. Chem. Mater. 2011, 23, 2027.

[16] Jayaraman, S.; Aravindan, V.; Suresh Kumar, P.; Ling, W. C.; Ramakrishna, S.; Madhavi, S. ACS Appl. Mater. Interfaces 2014, 6, 8660 .

[17] Fei, L.; Xu, Y.; Wu, X.; Li, Y.; Xie, P.; Deng, S.; Smirnov, S.; Luo, H. Nanoscale 2013, 5, 11102.

[18] Jo, C.; Kim, Y.; Hwang, J.; Shim, J.; Chun, J.; Lee, J. Chem. Mater. 2014, 26, 3508.

[19] Aravindan, V.; Sundaramurthy, J.; Jain, A.; Kumar, P. S.; Ling, W. C.; Ramakrishna, S.; Srinivasan, M. P.; Madhavi, S. ChemSusChem 2014, 7, 1858 . 
[20] Cheng, Q.; Liang, J.; Zhu, Y.; Si, L.; Guo, C.; Qian, Y. J. Mater. Chem. A 2014, 2, 17258.

[21] Xie, H.; Park, K.-S.; Song, J.; Goodenough, J. B. Electrochem. Commun. 2012, 19, 135

[22] Cussen, E. J.; Yi, T. W. S. J. Solid State Chem. 2007, 180, 1832.

[23] Satish, R.; Aravindan, V.; Ling, W. C.; Goodenough, J. B.; Madhavi, S. Adv. Energy. Mater. 2014, 4, 1301715.

[24] Ren, T.; Zhuang, Q. C.; Hao, Y. W.; Cui, Y. L. Acta Chim. Sinica 2016, 74, 833 (in Chinese). (任粀, 庄全超, 郝玉婉, 崔永丽, 化学 学报, 2016, 74, 833.)

[25] Luo, F.; Zheng, J. Y.; Chu, G.; Liu, B. N.; Zhang, S. L.; Li, H.; Chen, L. Q. Acta Chim. Sinica 2015, 73, 808 (in Chinese). (罗飞, 郑杰允, 褚春, 刘柏男, 张素林, 李泓, 陈立泉, 化学学报, 2015, 73, 808.)

[26] Lv, Z. Y.; Feng, R.; Zhao, J.; Fan, H.; Xu, D.; Wu, Q.; Yang, L. J.; Chen, Q.; Wang, X. Z.; Hu, Z. Acta Chim Sinica 2015, 73, 1013 (in Chinese). (吕之阳, 冯瑞, 赵进, 范豪, 徐丹, 吴强, 杨立军, 陈 强, 王喜章, 胡征, 化学学报, 2015, 73, 1013.)

[27] Qiu, Z. P.; Zhang, Y. J.; Xia, S. B.; Dong, P. Acta Chim Sinica 2015, 73, 992 (in Chinese). (邱振平, 张英杰, 夏书标, 董鹏, 化学学报, 2015, 73, 992.)

[28] Aravindan, V.; Ling, W. C.; Hartung, S.; Bucher, N.; Madhavi, S. Chem. Asian J. 2014, 9, 878.

[29] Luo, J.-Y.; Cui, W.-J.; He, P.; Xia, Y.-Y. Nat. Chem. 2010, 2, 760.

[30] Arun, N.; Aravindan, V.; Ling, W. C.; Madhavi, S. J. Alloys Compd. 2014, 603, 48.

[31] Gong, Z.; Yang, Y. Energy Environ. Sci. 2011, 4, 3223.

[32] Masquelier, C.; Croguennec, L. Chem. Rev. 2013, 113, 6552.

[33] Goodenough, J. B.; Kim, Y. Chem. Mater. 2009, $22,587$.

[34] Son, J. N.; Kim, S. H.; Kim, M. C.; Kim, G. J.; Aravindan, V.; Lee, Y. G.; Lee, Y. S. Electrochim. Acta 2013, 97, 210.

[35] Son, J. N.; Kim, S. H.; Kim, M. C.; Kim, K. J.; Aravindan, V.; Cho, W. I.; Lee, Y. S. J Appl. Electrochem. 2013, 4, 583.
[36] Cho, A. R.; Son, J. N.; Aravindan, V.; Kim, H.; Kang, K. S.; Yoon, W. S.; Kim, W. S.; Lee, Y. S. J. Mater. Chem. 2012, 22, 6556.

[37] Son, J. N.; Kim, G. J.; Kim, M. C.; Kim, S. H.; Aravindan, V.; Lee, Y. G.; Lee, Y. S. J. Electrochem. Soc. 2013, 160, A87.

[38] Wu, Z. S.; Zhou, G. M.; Yin, L. C.; Ren, W. C.; Li, F.; Cheng, H. M. Nano Energy 2012, 1, 107.

[39] Zhu, J.; Zhang, G. H.; Yu, X. Z.; Li, Q. H.; Lu, B.; Xu, Z. Nano Energy 2014, 3, 80 .

[40] Shen, B.; Zhai, W. T.; Zheng, W. Adv. Funct. Mater. 2014, 24, 4542.

[41] Shu, K. W.; Wang, C. Y.; Wang, M.; Zhao, C.; Wallace, G. G. J. Mater. Chem. A 2014, 2, 1325.

[42] Xu, W.; Xie, Z.; Cui, X.; Zhao, K.; Zhang, L.; Dietrich, G.; Dooley, K. M.; Wang, Y. ACS Appl. Mater. Interfaces 2015, 7, 22533.

[43] Park, G. D.; Kang, Y. C. Chem. Eur. J. 2015, 21, 9179.

[44] Mo, R.; Lei, Z.; Sun, K.; Rooney, D. Adv. Mater. 2014, 26, 2084.

[45] Guan, X.; Nai, J.; Zhang, Y.; Wang, P.; Yang, J.; Zheng, L.; Zhang, J.; Guo, L. Chem. Mater. 2014, 26, 5958.

[46] Kong, D. Z.; Luo, J. S.; Wang, Y. L.; Ren, W. N.; Yu, T.; Luo, Y. S.; Yang, Y. P.; Cheng, C. W. Adv. Funct. Mater. 2014, 24, 3815.

[47] Yin, L.; Zhang, Z.; Li, Z.; Hao, F.; Li, Q.; Wang, C.; Fan, R.; Qi, Y. Adv. Funct. Mater. 2014, 24, 4176

[48] Zhou, G.; Wang, D.; Li, F.; Zhang, L.; Li, N.; Wu, Z.; Wen, L.; Lu, G. Q.; Cheng, H. Chem. Mater. 2010, 22, 5306.

[49] Taberna, P. L.; Mitra, S.; Poizot, P.; Simon, P.; Tarascon, J. M. Nat. Mater. 2006, 5, 567.

[50] Zhou, J.; Song, H.; Ma, L.; Chen, X. RSC Adv. 2011, 1, 782.

[51] Zhan, L.; Wang, S.; Ding, L.-X.; Li, Z.; Wang, H. J. Mater. Chem. A 2015, 3, 19711 .

[52] Chen, M.; Xia, X.; Qi, M.; Yuan, J.; Yin, J.; Chen, Q. Mater. Res. Bull. 2016, 73, 125.

[53] Jena, A.; Penki, T. R.; Munichandraiah, N.; Shivashankar, S. A. J. Electroanal. Chem. 2016, 761, 21. 